\title{
Pylephlebitis and Liver Abscess Complicating Diverticulitis
}

\author{
Larrey $\mathbf{E}^{\mathbf{1}}$, Chassang $\mathbf{M}^{* 2}$, Antunes $\mathbf{0}^{1}$ and Anty $\mathbf{R}^{1,3,4}$ \\ ${ }^{1}$ University Hospital of Nice, Digestive Center, France \\ ${ }^{2}$ University Hospital of Nice, Medical Imaging Department, France \\ ${ }^{3}$ French National Institute of Health and Medical Research, France \\ ${ }^{4}$ University of Nice-Côte d'Azur, Faculty of Medicine, France
}

Received: August 02, 2018; Published: August 10,2018

*Corresponding author: Chassang Madlee, Archet Hospital, University Hospital of Nice, Medical Imaging Department, 151 route de Saint Antoine de Ginestière, Nice 06200, France

\begin{abstract}
Background: Sigmoid diverticulitis is a common abdominal disease that may require emergency treatment. The prognosis depends on the potentially dangerous complications, in particular pylephlebitis and hepatic abscess.

Case Report: We report the case of a patient with sigmoid diverticulitis, due to the ingestion of a foreign body, impacted in a diverticulum, complicated by pylephlebitis and hepatic abscess. The patient was treated by endoscopic extraction of the foreign body, antibiotic therapy and anticoagulation. Evolution was favorable.
\end{abstract}

Keywords: Diverticulitis; Pylephelbitis; Foreign Body; Liver Abscess; CT-Scan

\section{Background}

Sigmoid diverticulitis is one of the most common abdominal diseases requiring emergency treatment in Western countries. Conservative medical treatment often leads to the regression of symptoms, but some complications can be life threatening. Septic dissemination by contiguity (peri-intestinal abscesses/ generalized peritonitis) or hematogenous (pylephlebitis/hepatic abscess) may occur [1]. The suspicion of complications is based on clinical signs and symptoms (hyperthermia, abdominal pain, sometimes the presence of a palpable abdominal mass), and on blood tests showing an inflammatory response. However, the gold standard for the diagnosis is abdomino-pelvic computed tomography (CT) with injection of an iodinated contrast agent. A CT-scan is superior to ultrasound and magnetic resonance imagery for the visualization of deep intra-abdominal abscesses and septic thromboses due to its better spatial resolution and its greater acquisition range [2]. The treatment of these complications can be complex, and require a multidisciplinary approach, involving gastroenterologists, radiologists, endoscopists and surgeons. We report the case of a 69-yearold patient admitted to our hospital for sigmoid diverticulitis secondary to the impaction of an intra-colic foreign body, complicated by pylephlebitis and hepatic abscess, treated by interventional endoscopy, antibiotic therapy and anticoagulation.

\section{Case Report}

A 69-year-old Caucasian patient, with no previous medical history, presented to the emergency department for diffuse abdominal pain with nausea, vomiting, and fever. Blood tests showed an inflammatory syndrome (C-reactive protein , 400mg/L; leucocytosis, 13.93G/L; neutrophils, 12.62 G/L). An abdominal and pelvic CT with i.v injection of iodinated contrast media was performed. CT imaging showed numerous sigmoid diverticula with inflammatory thickening of the digestive walls in favor of sigmoid diverticulitis, associated with a $5 \mathrm{~cm}$ intra-luminal calcified linear foreign body (Figure 1). A hypodense 29-mm nodule of the VIth hepatic segment with inflammatory peripheral enhancement suggestive of a hepatic abscess was also found associated with segmental portal thrombosis of the same area (Figure 2). The diagnosis of an intra-sigmoidal foreign body complicated by sigmoid diverticulitis, which was further complicated by pylephlebitis and a single hepatic abscess was made. The initial treatment consisted of broad-spectrum antibiotic therapy, and colonoscopy, to extract the foreign body (a chicken bone) impacted in the mucosa by means of a "rat tooth clamp" (Rat tooth forceps). Mobilization of the foreign body revealed an underlying phlegmon (Figure 3). The patient confirmed accidental chicken bone ingestion a few days before. 
Evolution was favorable. Antibiotic therapy was administered for a total duration of 4 weeks and anticoagulation for 3 months.

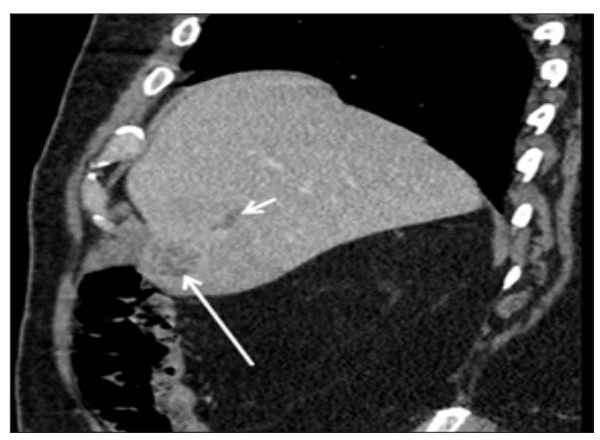

Figure 1: Abdomino-pelvic contrast enhanced CT scan (sagittal reconstruction) showing a lobulated hypo-dense nodule of the hepatic VIth segment diagnosed as a hepatic abscess (big arrow), associated with septic segmental portal thrombosis (small arrow).

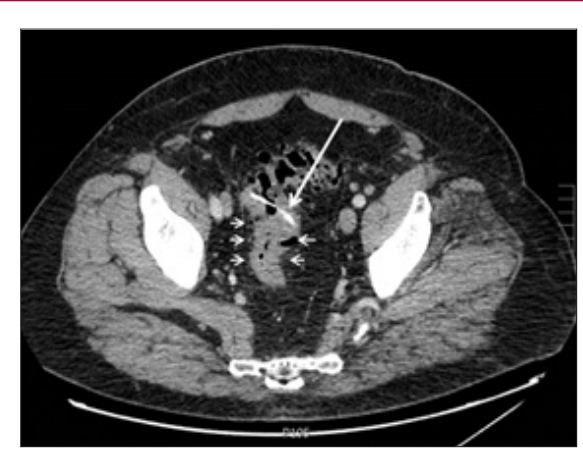

Figure 2: Abdomino-pelvic contrast enhanced CT scan (axial reconstruction) at a portal phase showing a calcified linear foreign body (big arrow) associated with inflammatory thickening of the diverticular sigmoidal walls (small arrows).

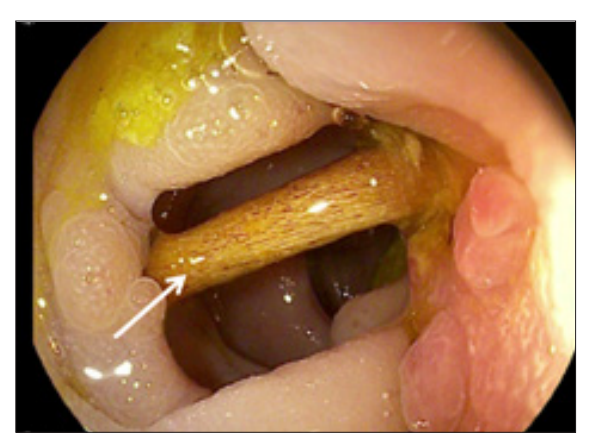

Figure 3: Photo of the colonoscopy (Pentax 14.65/3.8) showing the foreign body, a bone (arrow), impacted in a diverticulum with an associated local phlegmon.

\section{Discussion}

The originality of the reported case lies in the number of rare complications resulting from an intra-sigmoidal foreign body. These complications are sometimes fatal, even since the advent of effective antibiotics. This is the first reported case of a sigmoid foreign body complicated by diverticulitis associated with pylephlebitis and hepatic abscess. About 10 to $25 \%$ of patients with sigmoid diverticulosis develop diverticulitis [3]. The pathophysiology of diverticulitis includes acute inflammation of the diverticulum, followed by phlegmon and perforation [2]. Diverticulitis secondary to an intra-colic foreign body has been reported only in a few casesreport studies [4-5]. In this situation, endoscopic extraction of the foreign body is essential. Surgical resection may be sometimes necessary, especially when both extremities of the foreign body are impacted in the diverticulum and endoscopic extraction is not possible [4].

Abdomino-pelvic CT scanning with injection of iodinated contrast media, in addition to locating the foreign body if it is radiopaque, allows the detection of complications: pneumoperitoneum in the case of colonic perforation, signs of peritonitis, the presence of a pericolic abscess, or signs of distant dissemination of the infection [2]. Pylephlebitis or purulent thrombosis of the venous system usually results from gradual extension of suppurative thrombophlebitis secondary to an abdominal infection. However, sigmoid diverticulitis is a common cause of pylephlebitis. Pylephlebitis is a rare complication of diverticulitis: it is found in only $3 \%$ of cases [6]. However, in the 1990s it represented a serious condition with a mortality rate of more than $30 \%$ [7]. Most frequent etiologies were sigmoid diverticulitis (30\%), appendicitis (19\%) and inflammatory bowel disease (6\%) [8]. A recent meta-analysis published in 2016 found acute pancreatitis to be the most frequent cause, in $31 \%$ of cases, followed by sigmoid diverticulitis and appendicitis (19 and $2 \%$, respectively) [9].

This epidemiological change is probably due to the better efficacy of antibiotics and to the rapid treatment onset in the case of appendicitis and sigmoid diverticulitis. However, antibiotic treatment is started with more caution in patients with acute pancreatitis. Hepatic abscess is the main complication in the acute phase of pylephlebite, by dissemination of intra-hepatic septic emboli. The microbiology of the infection is often poly-microbial, and the two most frequent germs are Bacteroides fragilis and Escherichia coli [10]. The extension of thrombosis is a subsequent possible complication, which may be responsible for mesenteric ischemia [11]. Thrombosis may become chronic in a significant percentage of patients $(>10 \%)$ and may cause secondary intrahepatic portal hypertension without hepatocellular insufficiency [9]. Due to the low prevalence of this disease the treatment of pylephlebitis is not standardized and no international recommendations exist. Etiological treatment is a priority to annihilate the bacterial discharge in the portal blood system: the extraction of the foreign body in our case. Antibiotic therapy is also indicated, for a minimum duration of 4 weeks [7], with adaptation to the results of the bacterial cultures, knowing that the blood cultures are positive in less than $50 \%$ of patients [9].

The use of anticoagulants is still under debate, as is the total duration of treatment. Some authors advocate the use of curative anticoagulation only in patients with hemostatic disorders, including a protein S deficiency [12]. According to other authors, anticoagulation may reduce the risk of hepatic abscess by decreasing the risk of septic emboli [7]. The same authors found a mortality rate 
of $0 \%$ in patients receiving curative anticoagulation compared with $40 \%$ in non-anticoagulated patients [7]. More recent data are also in favor of anticoagulant treatment at a curative dose for a period of 3 to 6 months, which may be adapted according to the results of imaging examinations. The anticoagulation may reduce the risk of the extension of the thrombosis, in particular to the mesenteric veins, preventing mesenteric ischemia, which is an extremely severe complication [11]. Early use of anticoagulation may also allow a faster venous re-permeabilization and the diminution of the risk of secondary intra-hepatic portal hypertension [8]. For the hepatic abscesses, radiological drainage, in combination with appropriate antibiotic therapy, is recommended if the diameter is greater than $3 \mathrm{~cm}$ [13].

\section{Conclusion}

In conclusion, it is important for the clinicians and for the radiologists to recognize certain infrequent complications of diverticulitis, such as pylephlebitis. Indeed, early diagnosis is essential to start optimal treatment, because the latter may cause multiple intra-hepatic abscesses and mesenteric ischemia in the case of extension of thrombosis.

\section{References}

1. Wittmann DH, Schein M, Condon RE (1996) Management of secondary peritonitis. Ann Surg 224(1): 10-18.

2. Onur M, Akpinar E, Karaosmanoglu A, Isayev C, Karcaaltincaba M (2017) Diverticulitis: a comprehensive review with usual and unusual complications. Insights imaging 8(1): 19-27.

ISSN: 2574-1241

DOI: 10.26717/BJSTR.2018.07.001561

Chassang Madlee. Biomed J Sci \& Tech Res

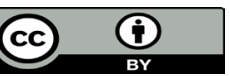

This work is licensed under Creative Commons Attribution 4.0 License

Submission Link: https://biomedres.us/submit-manuscript.php
3. Ferzoco LB, Raptopoulos V, SilenW (1998) Acute Diverticulis. N Engl J Med338(21): 1521-1526.

4. Lubel J, Wiley M (2005) Images of interest. Gastrointestinal: Foreign bodies and diverticulitis. Journal of gastroenterology and hepatology 20: 649 .

5. Bleich LM (2013) What's in her pocket: worsening diverticulitis. Am J Med 126(7): 581-582.

6. Falkowski A, Cathomas G, Zerz A, Rasch H, Tarr PE (2014) Pylephlebitis of a variant mesenteric vein complicating sigmoid diverticulitis. Radiology Case 8(2): 37-45.

7. Plemmons RM, Dooley DP, Longfield RN (1995) Septic Thrombophlebitis of the Portal Vein (Pylephlebitis): Diagnosis and Management in the Modern Era. Clinical Infectious Diseases 21(5): 1114-1120.

8. Kanellopoulou T, Alexopoulou A, Theodossiades G, Koskinas J, Archimandritis AJ (2010) Pylephlebitis: an overview of non-cirrhotic cases and factors related to outcome. Scand J Infect Dis 42(11-12): 804811.

9. Choudhry AJ, Baghdadi YM, Amr MA, Alzghari MJ, Jenkins DH, et al. (2016) Pylephlebitis: review of 95 cases. J Gastrointest Surg 20(3): 656661.

10. Kasper DL, Sahani D, Misdraji J (2005) N Engl J Med 353:713-722.

11. Lee BK, Ryu HH (2012) A case of pylephlebitis secondary to cecal diverticulitis. The J of Emergency Medicine 42(4): 81-85.

12. Baril N, Wren N, Radin R, Ralls P, Stain S (1996) The role of anticoagulation in pylephlebitis. Am J surg 172(5): 449-453.

13. Balthazar EJ, Gollapudi P (2000) Septic thrombophlebitis of the mesenteric and portal veins: CT imaging. J Comput Assist Tomogr 24(5): 755.

$\begin{array}{ll}\text { BIOMEDICAL } & \text { Assets of Publishing with us } \\ \text { RESEARCHES } & \text { - Global archiving of articles } \\ \text { - Immediate, unrestricted online access } & \text { - Rigorous Peer Review Process } \\ \text { https://biomedres.us/ }\end{array}$

